\title{
Morphometric study and occurrence of the palmaris longus muscle in human fetuses
}

\begin{abstract}
Introduction: The palmaris longus muscle has a short belly and a long tendon, it is located in the superficial layer of the anterior compartment of the forearm and usually has insertions in the medial humeral epicondyle and palmar aponeurosis. As it is an accessory muscle in wrist flexion, its absence does not cause functional impairment and its tendon is used as a graft in many surgical procedures. This study aimed to determine the occurrence of the palmaris longus muscle and its musculotendinous relations.
\end{abstract}

Methods: 20 forearms of 10 fetuses were dissected, with ages ranging from 19.6 to 37.8 weeks of gestation, belonging to the anatomy laboratory of the Federal University of Sergipe. The belly and tendon length of the palmaris longus muscle were measured using a $0.01 \mathrm{~mm}$ precision digital caliper.

Results: The palmaris longus muscle was present in all cases. The length of the palmaris longus muscle (PLM) was slightly longer on the right side, with its muscular belly being larger in the left dimidium and its tendon in the right. The relation between the length of the PLM and its tendon was slightly higher in the left dimidium; and the relation between the length of the MPL and its belly, as well as tendon/belly were bigger in the right dimidium.

Conclusion: The knowledge about the anatomy of the PLM may assist in the diagnosis of Carpal Tunnel Syndrome and Dupuytren's disease, as well as being useful to surgeons regarding tendon measurements to be used as grafts.

Keywords: palmaris longus muscle, tendon measurements, carpi ulnaris muscle, humerus
Volume 7 Issue I - 2020

\section{José Aderval Aragão, ${ }^{1,4}$ Julio Cezar de Oliveira Filho, ${ }^{2}$ Otávio Vinicius Nascimento de Oliveira, ${ }^{2}$ Layla Raíssa Dantas Souza, ${ }^{2}$ Bianca Holz Vieira, ${ }^{2}$ lapunira Catarina Sant'Anna Aragão, ${ }^{3}$ Felipe Matheus Sant'Anna Aragão, ${ }^{3}$ Francisco Prado Reis ${ }^{4}$}

'Department of Morphology, Federal University of Sergipe (UFS), Brazil

${ }^{2}$ Department of Medicine, Federal University of Sergipe (UFS), Brazil

${ }^{3}$ Medical School, University Center of Volta Redonda (UNIFOA), Brazil

${ }^{4}$ Medical School of Tiradentes University (UNIT), Brazil

Correspondence: José Aderval Aragão, Department of Morphology, Federal University of Sergipe, Marechal Rondon Avenue, São Cristóvão, Sergipe, Brazil, Tel +55-79-991916767, Email adervaluf@gmail.com

Received: December 23, 2019 | Published: January 07, 2020
Abbreviations: PLM, palmaris longus muscle; FCUM, flexor carpi ulnaris muscle; FCRM, flexor carpi radialis muscle; FDSM, flexor digitorum superficialis muscle; PTM, pronator teres muscle; PA, palmar aponeurosis; Redcircle, medial epicondyle of the humerus

\section{Introduction}

The palmaris longus muscle (PLM) is fusiform, slender and is located on the anterior face of the forearm, between the flexor carpi ulnaris and the flexor carpi radialis muscles, having proximal insertion into the medial epicondyle of the humerus and distally in the palmar aponeurosis. Phylogenetically, the PLM is considered a vestigial muscle in regression because it has a short belly and a long tendon ${ }^{1,2}$ and its function is considered insignificant in humans, ${ }^{3}$ although it weakly assists in flexing the wrist and resisting adduction and abduction movements. ${ }^{4}$ Numerous variations occur in the palmaris longus muscle and may even be absent unilaterally or bilaterally. The absence of the palmaris longus muscle seems to be hereditary, with dominant character, incomplete penetrance and lateral variability. ${ }^{2}$ When present, the PLM may present variations in the muscular belly and distal tendon. The muscular belly may be central (normal shape), distal (inverted shape), digastric or fully muscular. ${ }^{5}$ The distal tendon can be divided into two or three parts, that is, bitendinous or tritendinous, being referred to as an "accessory" muscle. ${ }^{6-11}$ The knowledge of the anatomical variations of the palmaris longus muscle is of great importance in the surgical area, since the PLM tendon meets the necessary length, diameter, availability and can be used in grafts without producing any functional deformity, ${ }^{12}$ for correction of upper eyelid ptosis, lower eyelid, lips and chins. ${ }^{3}$ The aim of the present study was to determine the frequency of the palmaris longus muscle, its morphometry and relations between its muscular and tendon parts.

\section{Material and methods}

20 upper limbs of 10 cadavers of human fetuses were dissected, with an average age of 30.36 weeks of gestation, being 10 right and 10 left, fixed and kept for more than one year in a $10 \%$ formaldehyde solution. The corpses belonged to the Anatomy Laboratory of the Federal University of Sergipe. The material was used in accordance with Law 8501 of November 30, 1992, which provides for the use of unclaimed corpses for study or scientific research purposes. Corpses with macroscopically detectable lower limb pathological changes were excluded. The observations resulting from anatomical dissection were documented through digital photography and measured using a $0.01 \mathrm{~mm}$ digital caliper.

\section{Results}

The palmaris longus muscle was present bilaterally in all dissected cadavers, without morphological variation of its muscular belly or tendon. Its muscle bundles were oriented converging toward the tendon. Its proximal insertion was through a common flexor tendon in the medial epicondyle of the humerus, where the muscle belly had a medial relation with the flexor carpi ulnaris muscle and laterally with the flexor carpi radialis muscle that posteriorly covered part of the flexor digitorum superficialis muscle and distally his tendon continued with the palmar aponeurosis (Figure 1). The PLM morphometry according to the dimidium, as well as its muscular and tendon relation, are shown in Table $1 \& 2$. 


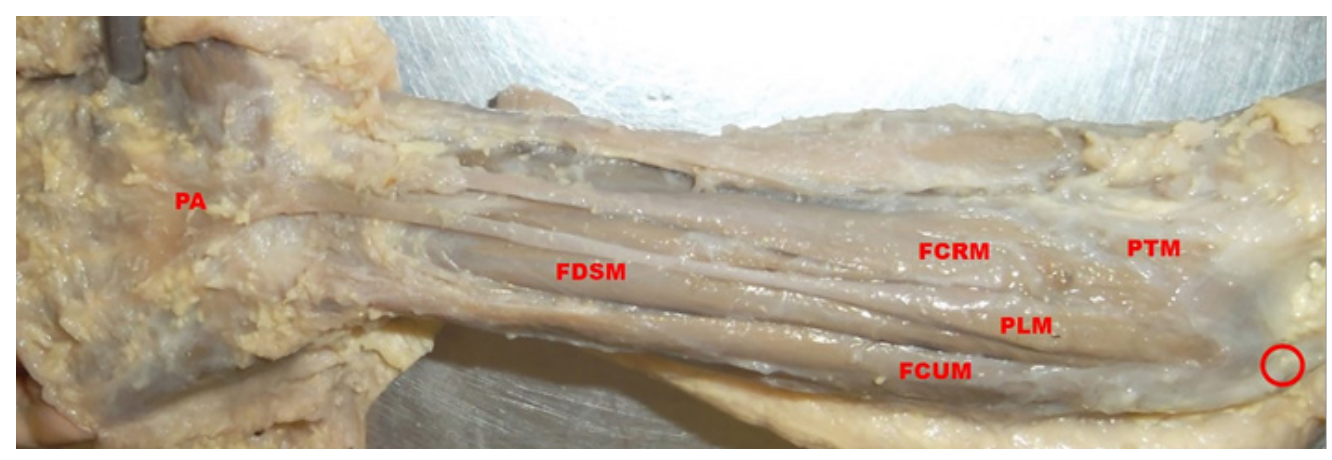

Figure I The palmaris longus muscle.

PLM, palmaris longus muscle; FCUM, flexor carpi ulnaris muscle; FCRM, flexor carpi radialis muscle; FDSM, flexor digitorum superficialis muscle; PTM, pronator teres muscle; PA, palmar aponeurosis; Redcircle, medial epicondyle of the humerus.

Table I Palmaris longus muscle morphometry $(\mathrm{mm})$

\begin{tabular}{llc}
\hline & \multicolumn{2}{c}{ Dimidium } \\
\cline { 2 - 3 } Average length $(\mathbf{m m})$ & Right & Left \\
\hline Muscular belly & 22.1 & 23.8 \\
Tendon & 35.9 & 33.0 \\
Total & 58.0 & 56.8 \\
\hline
\end{tabular}

mm, millimeters

Table 2 Morphometric relation muscle, belly and tendon

\begin{tabular}{lll}
\hline & \multicolumn{2}{c}{ Dimidium } \\
\cline { 2 - 3 } Relation & Right & Left \\
\hline Muscle/tendon & 1.61 & 1.72 \\
Muscle/belly & 2.62 & 2.38 \\
Tendon/belly & 1.62 & 1.38 \\
\hline
\end{tabular}

\section{Discussion}

The presence of the PLM has been studied predominantly through the method of dissection in cadavers or by inspection in living subjects. In our study, the PLM was present in $100 \%$ of the dissected cases, unlike Olewnik et al. ${ }^{13}$ who found that the PLM was present in $62.5 \%$ of fetuses. Its proximal and distal insertion is in accordance with what is reported in the anatomy textbooks, ${ }^{4,14}$ that is, the proximal insertion is in the medial epicondyle of the humerus and distally in the palmar aponeurosis. Few studies have reported the PLM morphometry in human fetuses, in our study the average length of the PLM and its tendon of fetuses aged from 19.6 to 37.8 weeks of gestation was of 30.36 , our results were larger than those found by Olewnik et al. ${ }^{13}$ who studied 160 limbs of fetuses aged 18 to 38 weeks of gestation which had an average length of $25.9 \mathrm{~mm}$, as well as what was found by Kocabiyik et al. ${ }^{15}$ who studied 44 limbs of fetuses, with an average length of $25.9 \mathrm{~mm}$ long at 17 to 40 weeks of gestation.

The most common anatomical anomaly in the PLM literature is its agenesis, ${ }^{16,17}$ but studies conducted in different populations show different values, with high prevalence in Turks $(63.9 \%)$ and Caucasians (25\%) and low in Asians (4, 8\%), blacks (3\%), and Koreans $(0.6 \%) .{ }^{18-23}$ These studies reinforce the idea that the absence of the PLM depends on the population, although being, not so clearly, related to genetic factors. In our study, no bilateral or unilateral absence of the PLM was found. However, in the literature, there is disagreement regarding its absence and whether it is more common in women and in the left dimidium. ${ }^{16,18,24,25}$ It is also worth noting that there was no variation in the shape of the tendon or the muscle belly, that is, no distal muscle belly (inverted shape), digastric or fully muscular were found, as reported by Reimann et al. ${ }^{5}$ nor the distal tendon bitendinosus or tritendinosus were identified in several studies..$^{6-11,15,26}$ It is worth noting that the PLM fulfills a small contribution function of the wrist flexion, so that its absence does not compromise such functionality. ${ }^{20}$ Thus, the palmaris longus muscle has considerable clinical relevance. Variations of the PLM anatomy are generally believed to be asymptomatic; however, several authors have described that the PLM inversion could cause symptomatic compression of the median nerve. ${ }^{27-30}$ Knowing this variation, we could avoid unsuccessful surgical explorations in the diagnosis of conditions such as carpal tunnel syndrome ${ }^{3}$ and Dupuytren's disease, which is a pathological condition with fibrous degeneration in the longitudinal superficial layer of the palmar aponeurosis. ${ }^{31}$ In addition, the PLM tendon is used as a landmark to locate the underlying median nerve for surgical procedures, ${ }^{4}$ as well as being used as a graft in a large number of surgical procedures, such as chronic flexor tendon injuries, ligament reconstruction, pulley reconstruction, eye defects, blepharopitosis and other surgical reconstructions. ${ }^{22}$ Thus, the knowledge of their varied forms, measurements and occurrence in different populations is of great importance.

\section{Conclusion}

The palmaris longus muscle is functionally irrelevant in humans, however its knowledge may help in the understanding of various pathological processes and improve success rates in reconstructive surgery using the tendon graft. Surgeons should be familiar with potential anatomical variations, as well as tendon and muscle belly 
measurements in the local population. Therefore, the findings of this study may be useful in providing anatomical knowledge to clinicians and surgeons, especially plastic surgeons in surgical procedures, since the tendon / belly ratio was higher on the right side in the current study and thus may become an initial option for grafts.

\section{Acknowledgments}

None.

\section{Conflicts of interest}

Author declares there are no conflicts of interest.

\section{References}

1. Koo CC, Roberts AH. The palmaris longus tendon. Another variation in its anatomy. J Hand Surg Br. 1997;22(1):138-139.

2. Thompson JW, McBatts J, Danforth $\mathrm{CH}$. Hereditary and racial variation in the musculus palmaris longus. American Journal of Physical Anthropology. 1921;4(2):205-218.

3. Park MJ, Namdari S, Yao J. Anatomic variations of the palmaris longus muscle. Am J Orthop (Belle Mead NJ). 2010;39(2):89-94.

4. Standring S. Gray's Anatomy. 39th ed. London: Churchill Livingstone. 2007:995-1027.

5. Reimann AF, Daseler EH, Anson BJ, et al. The palmaris longus muscle and tendon. A study of 1600 extremities. Anat Rec. 1944;89(4):495-505.

6. Roberts PH. An anomalous accessory palmaris longus muscle. Hand. 1972;4(1):40-41.

7. Schlafly B, Lister G. Median nerve compression secondary to bifid reversed palmaris longus. J Hand Surg Am. 1987;12(3):371-373.

8. Rubino C, Paolini G, Carlesimo B. Accessory slip of the palmaris longus muscle. Ann Plast Surg. 1995;35(6):657-659.

9. Celik HH, Sargon MF, Yilmaz E, et al. Bitendinous palmaris longus muscle. Bull Assoc Anat (Nancy). 1996;80(250):15-16.

10. Kawashima T, Kikushima S, Yokota E, et al. A case of an accessory palmaris longus muscle and a duplicate palmaris longus muscle with special reference to their nerve supply-morphologic significance of a common innervation trunk. Okajimas Folia Anat Jpn. 2002;79(2-3):7581.

11. Tiengo C, Macchi V, Stecco C, et al. Epifascial accessory palmaris longus muscle. Clin Anat. 2006;19(6):554-557.

12. Troha F, Baibak GJ, Kelleher JC. Frequency of the palmaris longus tendon in North American caucasians. Ann Plast Surg. 1990;25(6):477478.

13. Olewnik $Ł$, Waśniewska A, Polguj M, et al. Morphological variability of the palmaris longus muscle in human fetuses. Surg Radiol Anat. 2018;40(11):1283-1291.

14. Moore KL, Dalley AF, Agur AM. Anatomia Orientada para a Clínica. 7th ed. Guanabara Koogan LTDA. 2014;743-744.
15. Kocabiyik N, Yildiz S, Develi S, et al. Morphometric analysis of the palmaris longus muscle: a fetal study Morphometric analysis of the palmaris longus muscle: a fetal study. Int J Exp Clin Anat. 2014;6(12):953-957.

16. Sebastin SJ, Puhaindran ME, Lim AY, et al. The prevalence of absence of the palmaris longus-a study in a Chinese population and a review of the literature. J Hand Surg. 2005;30(5):525-527.

17. Kyung D, Lee J, Choi I, et al. Different frequency of the absence of the palmaris longus according to assessment methods in a Korean population. Anat Cell Biol. 2012;45(1):53-56.

18. Ceyhan O, Mavt A. Distribution of agenesis of palmaris longus muscle in 12 to 18 years old age groups. Indian J Med Sci. 1997;51(5):156-160.

19. Thompson NW, Mockford BJ, Cran GW. Absence of the palmaris longus muscle: a population study. Ulster Med J. 2001;70(1):22-24.

20. Sebastin SJ, Lim AY. Clinical assessment of absence of the palmaris longus and its association with other anatomical anomalies-- a Chinese population study. Ann Acad Med Singapore. 2006;35(4):249-253.

21. Kigera JW, Mukwaya S. Frequency of agenesis Palmaris longus through clinical examination--an East African study. PLoS One. 2011;6(12):e28997.

22. Angelini Júnior LC, Angelini FB, Oliveira BC, et al. Use of the tendon of the palmaris longus muscle in surgical procedures: study on cadavers. Acta Ortop Bras. 2012;20(4):226-229.

23. Ioannis D, Anastasios K, Konstantinos N, et al. Palmaris longus muscle's prevalence in different nations and interesting anatomical variations: review of the literature. J Clin Med Res. 2015;7(11):825-830.

24. Mathew AJ, Sukumaran TT, Joseph S. Versatile but temperamental: a morphological study of palmaris longus in the cadaver. J Clin Diagn Res. 2015;9(2):AC01-AC03.

25. Olewnik $Ł$, Wysiadecki G, Polguj M, et al. Anatomical variations of the palmaris longus muscle including its relation to the median nerve - a proposal for a new classification. BMC Musculoskelet Disord. 2017;18(1):539.

26. Albay S, Kastamoni Y, Sakalli B, et al. Anatomy and variations of palmaris longus in fetuses. Rom J Morphol Embryol. 2013;54(1):85-89.

27. Twoon M, Jones CD, Foley J, et al. Reversed palmaris longus muscle: a report of two cases. Case Reports Plast Surg Hand Surg. 2017;4(1):7376.

28. Getzmann JM, Schweizer A. Surgical resection of a reversed palmaris longus muscle causing chronic exertional compartment syndrome in a competitive swimmer: a case report. Orthop $J$ Sports Med. 2018;6(5):2325967118769615.

29. George SC, Hassan Z. A bifid reversed palmaris longus muscle causing median nerve compression in a child. J Hand Surg Eur Vol. 2018;43(6):665-666

30. Longhurst G, Stone D, Mahony N. Bilateral reversed palmaris longus muscle: a case report and systematic literature review. Surg Radiol Anat 2019.

31. Dupuytren G. Clinical lectures on surgery. Lancet. 1834;23(558):56-59. 